\title{
Influence of optical material properties on strong coupling in organic semiconductor based microcavities
}

Laura Tropf, Christof P. Dietrich, Stefanie Herbst, Alexander L. Kanibolotsky, Peter J. Skabara, Frank Würthner, Ifor D. W. Samuel, Malte C. Gather, and Sven Höfling

Citation: Appl. Phys. Lett. 110, 153302 (2017); doi: 10.1063/1.4978646

View online: https://doi.org/10.1063/1.4978646

View Table of Contents: http://aip.scitation.org/toc/apl/110/15

Published by the American Institute of Physics

\section{Articles you may be interested in}

Three-dimensional photonic confinement in imprinted liquid crystalline pillar microcavities

Applied Physics Letters 110, 201113 (2017); 10.1063/1.4983565

Optical probing of the Coulomb interactions of an electrically pumped polariton condensate Applied Physics Letters 110, 151103 (2017); 10.1063/1.4979836

Observation of subwavelength localization of cavity plasmons induced by ultra-strong exciton coupling Applied Physics Letters 110, 171101 (2017); 10.1063/1.4979838

Complex permittivity analysis revisited: Microwave spectroscopy of organic semiconductors with resonant cavity Applied Physics Letters 110, 153303 (2017); 10.1063/1.4980078

A method for direct contact resistance evaluation in low voltage coplanar organic field-effect transistors Applied Physics Letters 110, 153304 (2017); 10.1063/1.4980069

Independent tuning of excitonic emission energy and decay time in single semiconductor quantum dots Applied Physics Letters 110, 151102 (2017); 10.1063/1.4979481

\section{Scilight} Sharp, quick summaries illuminating the latest physics research

\section{Sign up for FREE!}

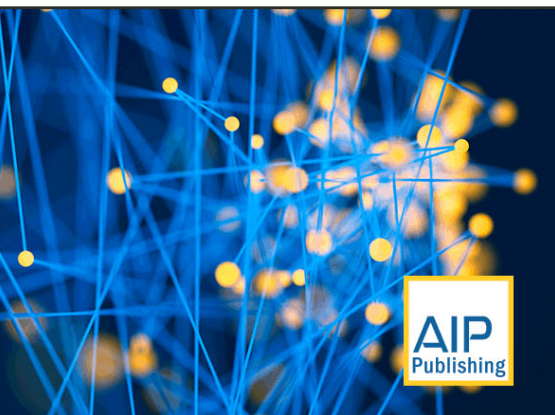




\title{
Influence of optical material properties on strong coupling in organic semiconductor based microcavities
}

\author{
Laura Tropf, ${ }^{1}$ Christof P. Dietrich, ${ }^{1,2}$ Stefanie Herbst, ${ }^{3}$ Alexander L. Kanibolotsky, ${ }^{4}$ \\ Peter J. Skabara, ${ }^{4}$ Frank Würthner, ${ }^{3}$ Ifor D. W. Samuel, ${ }^{1}$ Malte C. Gather, ${ }^{1, a)}$ \\ and Sven Höfling ${ }^{1,2, a)}$ \\ ${ }^{1}$ Organic Semiconductor Centre, SUPA, School of Physics and Astronomy, University of St Andrews, \\ North Haugh, St Andrews, KY16 9SS, United Kingdom \\ ${ }^{2}$ Technische Physik, Universität Würzburg, Am Hubland, D-97074 Würzburg, Germany \\ ${ }^{3}$ Institut für Organische Chemie and Center for Nanosystems Chemistry, Universität Würzburg, Am Hubland, \\ D-97074 Würzburg, Germany \\ ${ }^{4}$ WestCHEM, Department of Pure and Applied Chemistry, University of Strathclyde, Glasgow, G1 1XL, \\ United Kingdom
}

(Received 27 September 2016; accepted 2 March 2017; published online 12 April 2017)

\begin{abstract}
The optical properties of organic semiconductors are generally characterised by a number of material specific parameters, including absorbance, photoluminescence quantum yield, Stokes shift, and molecular orientation. Here, we study four different organic semiconductors and compare their optical properties to the characteristics of the exciton-polaritons that are formed when these materials are introduced into metal-clad microcavities. We find that the strength of coupling between cavity photons and excitons is clearly correlated with the absorptivity of the material. In addition, we show that anisotropy strongly affects the characteristics of the formed exciton-polaritons. Published by AIP Publishing.

[http://dx.doi.org/10.1063/1.4978646]
\end{abstract}

Since the first observation of exciton-polaritons in a strongly coupled microcavity, ${ }^{1}$ planar microcavities have become a standard tool for studying their fundamental properties. The bosonic nature of polaritons allows for observation of Bose-Einstein condensation in a non-equilibrium system up to room temperature, ${ }^{2-6}$ and their non-linear properties give rise to a range of interesting physical properties, including bistability ${ }^{7}$ and bright and dark solitons. ${ }^{8-11}$ When polaritons undergo condensation within a microcavity, coherent light is emitted and the cavity acts as a polariton laser. Since polariton lasing does not require population inversion, it can have a lower threshold than conventional lasers. ${ }^{12}$

Polariton physics has mainly been studied using inorganic semiconductor heterostructures. Recently, however, organic semiconductors have received great attention. ${ }^{5,6,13-16,27}$ In particular, the potentially lower thresholds of polariton lasing compared to conventional lasing may open a path to realising an electrically driven organic laser. There are significant differences between polaritons in organic and inorganic materials, stemming from the fundamentally different properties of the involved excitons. Binding energies of excitons in organic materials exceed those in inorganic semiconductors by far (organic: $\sim 0.5 \mathrm{eV}$; inorganic: $\sim 1 \mathrm{meV}$ ). As a consequence, organic polaritons are more localised (socalled Frenkel excitons) and exhibit higher thermal stability than the polaritons in inorganic systems; they easily persist up to room temperature. ${ }^{13}$ Another key difference lies in the large dipole transition moments inherent to organic materials, resulting in a stronger coupling between excitons and the photon modes of a surrounding microcavity. This manifests itself in large Rabi splittings.

\footnotetext{
${ }^{\text {a) }}$ Authors to whom correspondence should be addressed. Electronic addresses: sven.hoefling@physik.uni-wuerzburg.de and mcg6@st-andrews.ac.uk
}

At the same time, the disorder present in organic materials results in relatively large linewidths of the excitonic resonance $(\sim 10$ to $1000 \mathrm{meV}$ in organic semiconductors compared to $\sim 1 \mathrm{meV}$ in inorganic materials). Strong coupling is crucially dependent on the excitonic and photonic lifetimes that lead to homogeneous line broadening. The impact of inhomogeneous broadening caused by the disorder, which dominates the spectral shape, is, however, not quite clear. The emissivity of weakly coupled organic materials depends on several material parameters, including the oscillator strength, the structural relaxation of an electronically excited molecule (Stokes shift, $\Delta_{\text {abs-em }}$ ), the competition of radiative and non-radiative processes (photoluminescence quantum yield, PLQY), and the molecular orientation in the film. These parameters vary strongly between different organic semiconductors and can to some extent be adjusted by tuning the chemical structure and the processing conditions of the material. Hence, their effect on strong coupling and on the emission from the polariton states is of great importance, both to improve general understanding of strong coupling in organic systems and to optimise the performance of future organic polariton lasers. We observed, however, that there is some uncertainty in the literature about which material properties are beneficial for achieving strong coupling. ${ }^{6,16-20}$

Here, we clarify the correlation between several optical material properties and the coupling strength by comparing the Rabi splitting for different organic semiconductors embedded in metal-clad microcavities with the optical properties of these emitters. Three of the tested materials were semiconducting polymers that were chosen because they are highly efficient organic laser materials with strong absorption in the blue to UV spectral range. ${ }^{21,22}$ The fourth material serves as a contrasting material that is based on a newly developed liquid-crystalline J-aggregate forming perylene bisimide dye called MEH-PBI. ${ }^{23}$ Thin films of MEH-PBI 

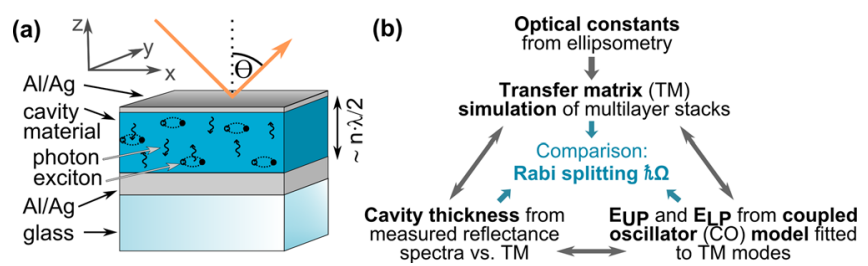

FIG. 1. (a) Schematic of the sample structure with the light path (orange) and (b) schematic of the procedure used for characterising the samples.

absorb in the red region of the spectrum and have a narrower linewidth and a smaller Stokes shift compared to polymer films due to J-aggregation. The spectral positions of the excitonic resonances of all investigated materials open a pathway towards hybrid organic-inorganic microcavities: the blue emitters can potentially be combined with wide-band-gap materials like InGaN or $\mathrm{ZnO}$, whereas the spectral characteristics of the J-aggregate material are compatible with AlGaAs or InGaP.

Comparing the organic microcavities, we find that while the Rabi splitting is closely linked to the absorption coefficient, correlations with other material properties like the Stokes shift or fluorescence quantum yield are not evident. Furthermore, we show that good agreement between simulated and measured reflectance data can only be obtained when taking into account the optical anisotropy of the materials used.

The cavities studied were comprised of two metal mirrors (Al or Ag) sandwiching the organic material (see Fig. 1(a)). The top mirror was thin enough to be semitransparent and thus allowed for optical probing of the cavity. For each emitter, the reflectance spectrum was recorded at a fixed angle of reflection, $\Theta$, for several cavities of systematically varied thickness.

The characterisation steps performed in this paper are summarised in Figure 1(b). First, the refractive index $n$ and the extinction coefficient $k$ of the materials were determined. These were then fed into a transfer matrix (TM) algorithm to simulate the reflectance of the sample (assuming perfectly parallel interfaces). Guided by these TM calculations, cavities with a range of organic film thicknesses were fabricated. The exact organic film thickness for each cavity was then determined by fitting TM calculations to measured reflectance spectra. The reflectance spectra showed two characteristic dips (referred to as modes in the following). The spectral position of each mode was extracted from the experimental data and collated with (i) the TM simulations and (ii) a coupled oscillator (CO) model (described below), yielding the Rabi splitting for each material.

We investigated the two homopolymers poly[9,9-dioctylfluorenyl-2,7-diyl] (PF8) and poly[2, 5-bis $\left(2^{\prime}, 5^{\prime}\right.$-bis ( $2^{\prime \prime}$-ethyl-hexyl-oxy)phenyl)-p-phenylene vinylene] (BBEHPPPV $){ }^{21}$ the copolymer poly(9,9-dioctylfluorene-alt-benzothiadiazole) (F8BT), and a J-aggregate forming dye (MEHPBI). Neat films of the desired thickness were formed by spin-coating from toluene (PF8, BBEHP-PPV and F8BT) and chloroform (MEH-PBI) solutions, respectively.

Figures 2(a)-2(d) show the real and imaginary parts of the complex refractive indices $\tilde{n}=n+i k$ of the investigated materials (determined by ellipsometry). All four materials show a strong anisotropy between in-plane $\left(n_{\mathrm{xy}}, k_{\mathrm{xy}}\right)$ and out-of-plane $\left(n_{\mathrm{z}}, k_{\mathrm{z}}\right)$ properties. In the polymers, this can be explained by the polymer chains tending to lie in the plane of the film and the dipoles being oriented along the backbone. ${ }^{24}$ This results in the absorption being much stronger for electric field vectors aligned in the plane than perpendicular to it (corresponding to waves propagating perpendicularly and parallel to the film surface, respectively). The main features of the optical constants are at the same spectral positions for inand out-of-plane directions, albeit weaker for $n_{z}$ and $k_{z}$. In (a) PF8

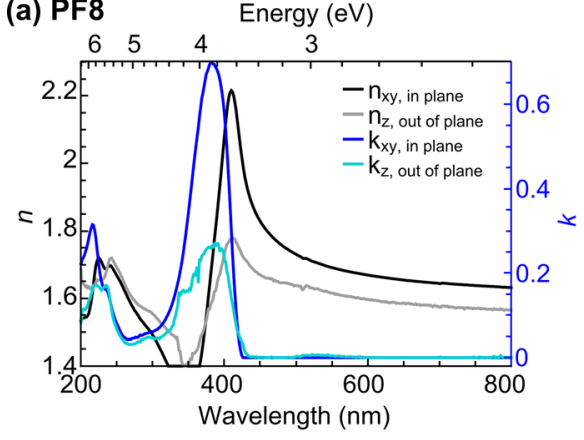

(c) F8BT

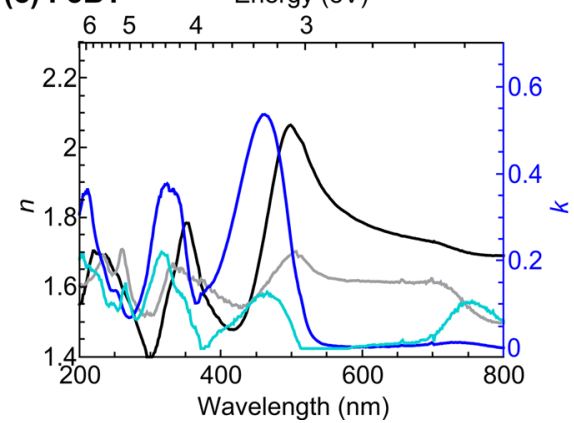

(b) BBEHP-PPV Energy (eV)

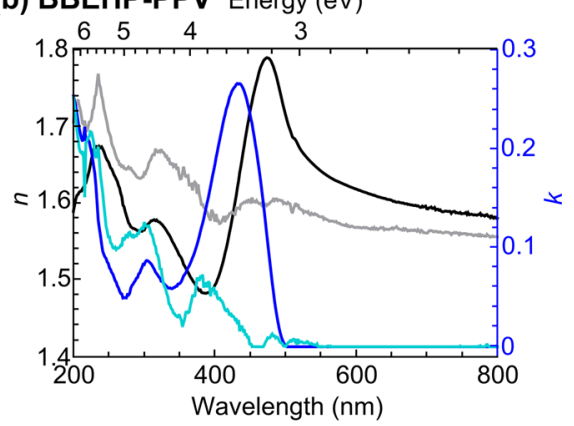

(d) MEH-PBI Energy (eV)

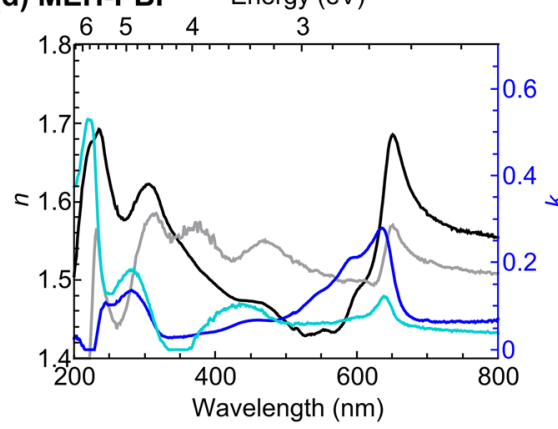

(e) Chemical Structures PF8
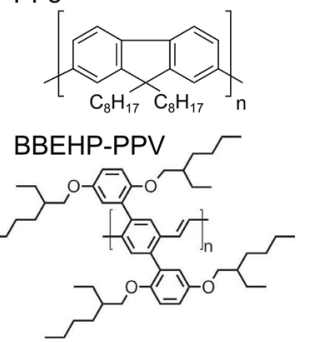

F8BT

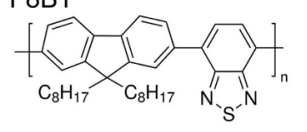

MEH-PBI

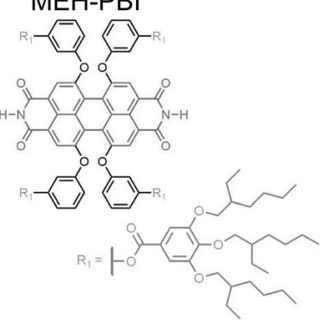

FIG. 2. (a)-(d) Optical constants (real and imaginary part of refractive index, $n$ and $k$, respectively) of the investigated organic materials: (a) PF8, (b) BBEHPPPV, ${ }^{21}$ (c) F8BT and (d) MEH-PBI. ${ }^{23}$ The legend of (a) refers to subfigures (a) to (d). (e) Corresponding chemical structures. 
TABLE I. Comparison of the optical properties of the investigated active materials to the observed Rabi splittings. For F8BT, $X_{1}$ and $X_{2}$ denote the two transitions observed. For all materials except for MEH-PBI, the values for the PLQY and radiative lifetime refer to literature values for solution-deposited neat films of the respective material.

\begin{tabular}{|c|c|c|c|c|c|c|c|c|}
\hline & $E_{\mathrm{X}}$ & FWHM & $\alpha_{\max }$ & $\alpha_{\text {int }}$ (a.u.) & PLQY & lifetime & $\Delta_{\text {abs-em }}$ & $\hbar \Omega$ \\
\hline PF8 & $3.27 \mathrm{eV}$ & $0.57 \mathrm{eV}$ & $1.6 \times 10^{5} \mathrm{~cm}^{-1}$ & 0.27 & $0.55^{25}$ & $227 \mathrm{ps}^{22}$ & $0.31 \mathrm{eV}$ & $1.09 \pm 0.05 \mathrm{eV}$ \\
\hline BBEHP-PPV & $2.84 \mathrm{eV}$ & $0.54 \mathrm{eV}$ & $0.8 \times 10^{5} \mathrm{~cm}^{-1}$ & 0.16 & $0.84^{26}$ & $650 \mathrm{ps}^{21}$ & $0.36 \mathrm{eV}$ & $0.61 \pm 0.05 \mathrm{eV}$ \\
\hline F8BT $X_{1}$ & $2.68 \mathrm{eV}$ & $0.50 \mathrm{eV}$ & $0.9 \times 10^{5} \mathrm{~cm}^{-1}$ & 0.17 & $0.58^{22}$ & $2028 \mathrm{ps}^{22}$ & $0.38 \mathrm{eV}$ & $0.84 \pm 0.05 \mathrm{eV}$ \\
\hline F8BT $X_{2}$ & $3.82 \mathrm{eV}$ & $0.58 \mathrm{eV}$ & $1.2 \times 10^{5} \mathrm{~cm}^{-1}$ & 0.21 & & & & $0.88 \pm 0.05 \mathrm{eV}$ \\
\hline MEH-PBI & $1.94 \mathrm{eV}$ & $0.09 \mathrm{eV}$ & $0.4 \times 10^{5} \mathrm{~cm}^{-1}$ & 0.07 & 0.13 & $4800 \mathrm{ps}$ & $0.04 \mathrm{eV}$ & $0.32 \pm 0.05 \mathrm{eV}$ \\
\hline
\end{tabular}

MEH-PBI, by contrast, the preferred absorption of in- and out-of-plane electric fields depends on the spectral region: the extinction coefficient at $\lambda=638 \mathrm{~nm}$ is far more pronounced in the plane, while the broad absorption around $\lambda=450 \mathrm{~nm}$ is strongly increased perpendicular to the film surface.

The spectral shape of the extinction peaks was studied by fitting them to multiple Gaussians since for the organic materials studied, it is dominated by inhomogeneous broadening due to disorder in the materials. The fits showed that the absorption peaks are comprised of several (two to three) vibronic modes. The FWHM-widths of these modes ranged from $0.07 \mathrm{eV}$ (J-aggregate peak of MEH-PBI) to $0.43 \mathrm{eV}$ (BBEHP-PPV). The Gaussian fits were used to determine the integrated absorption value $\left(\alpha_{\text {int }}\right)$ for each peak.

The optical properties of all investigated materials are summarised in Table I. Most parameters were measured inhouse. The PLQY and exciton lifetime of MEH-PBI were determined from a neat film using a Hamamatsu integrating sphere and a time-resolved photoluminescence setup, respectively. The PLQY and exciton lifetime listed for PF8, F8BT, and BBEHP-PPV represent literature values measured under comparable conditions. A comparison of these properties shows similar features in the polymers but more distinct properties for the J-aggregate. As expected, the latter has a considerably lower linewidth (values see above) and smaller $\Delta_{\text {abs-em }}$ than the polymers $(0.09 \mathrm{eV}$ and $0.04 \mathrm{eV}$ compared to $0.49 \pm 0.09 \mathrm{eV}$ and $0.35 \pm 0.04 \mathrm{eV}$, respectively). Furthermore, the thin film PLQY of MEH-PBI is much lower (13\%) than for the other polymers (literature values, ranging from $53 \%$ in $\mathrm{PF}^{25}$ to $84 \%$ in BBEHP-PPV ${ }^{26}$ ). The maximum absorption coefficient, $\alpha_{\max }$, is deduced from the peak extinction coefficient $k_{\max }$ as $\alpha_{\max }=4 \pi k_{\max } / \lambda_{0}$, where $\lambda_{0}$ is the spectral position of the peak extinction. The value of $\alpha_{\max }$ increases when going from MEH-PBI to BBEHP-PPV, F8BT and PF8 from $\alpha_{\max }$, MEH-PBI $=0.4 \times 10^{5} \mathrm{~cm}^{-1}$ to $\alpha_{\max }$, $\mathrm{PF} 8=1.6 \times 10^{5} \mathrm{~cm}^{-1}$, which corresponds to a four-fold increase. In contrast to the other materials, F8BT shows two excitonic resonances across the spectral range of interest.

To illustrate the origin of different features of the reflectance spectrum of our cavities, Figure 3 shows a comparison of an experimental spectrum of a MEH-PBI cavity with TM simulations of four different hypothetical sample structures (A to D). Of these, only Structure A, which takes the anisotropy of absorption and refractive index into account, reproduces the experimental data well. Here, both the position and the shape of the spectrum with its two reflectance minima are well described. If instead an isotropic cavity material of the same thickness is assumed (Structure B), the ratio of the mode depths, as well as their distance, differs significantly from the measured features. The polymers, which are preferentially aligned in the plane of the film, show the same effect, albeit to a smaller extent. This clearly shows the importance of taking the microscopic structure and molecular stacking into account.

The other simulated structures show examples of the expected reflectance of uncoupled cavities: Structure C assumes a constant refractive index $n_{\text {eff }}=1.6+0 \times i$ (no absorption). This corresponds to a cavity of the same optical thickness filled with a transparent material. We refer to Structure C as "empty" cavity and to the mode position as bare cavity mode $E_{\mathrm{C}}$. Structure D represents a stack without a top mirror, and its reflection is dominated by the bulk material absorption at the excitonic energy $E_{\mathrm{x}}$. Note that the positions of the dips in reflectance of Structures C and D are located at approximately the same wavelength, indicating a very small detuning of cavity mode and excitonic resonance, $E_{\mathrm{X}}-E_{\mathrm{C}}$, in this cavity structure.

The splitting of $E_{\mathrm{C}}$ and $E_{\mathrm{X}}$ into two modes for microcavities containing an active material is a strong indication that the two dips in the reflectance spectrum represent the upper and lower polariton modes, respectively.

To confirm this, we compare the spectral positions of the dips in the measured reflectance spectra for samples with different thicknesses to spectra simulated with the TM algorithm, taking into account the optical anisotropy of the materials (Structure A in Figure 3). Since the TM simulation and the experimental spectrum are interconnected through the TM fitting based thickness measurement, we also included

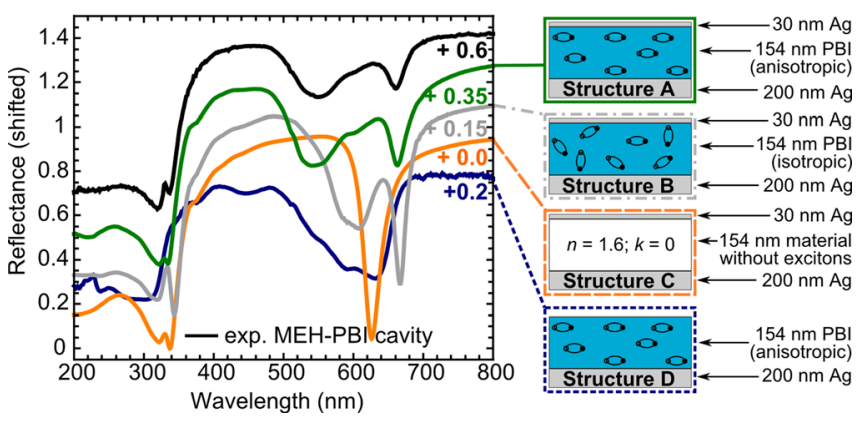

FIG. 3. Left: Comparison of the experimental reflectance spectrum of a Ag-mirrored MEH-PBI cavity (black solid line) to transfer matrix calculations of the cavity with $d_{\mathrm{MEH}-\mathrm{PBI}}=154 \mathrm{~nm}$ assuming anisotropic (Structure A, green, solid line) or isotropic (Structure B, grey, dash-dotted line) optical constants, an empty cavity with $n=n_{\mathrm{MEH}-\mathrm{PBI} \text {, eff }}=1.6=$ const. (Structure $\mathrm{C}$, orange, dashed line), and a thin film of MEH-PBI without a top mirror (Structure D, blue, dotted line). Spectra vertically shifted for clarity. Right: Schematic illustration of different cavity structures. 
an independent analytical coupled oscillator (CO) model for analysing the data further. Depending on the number of excitonic resonances, the model includes two- (BBEHP-PPV and MEH-PBI), three- (PF8), or four-level (F8BT) coupled oscillators. The eigenenergies of the polariton branches are given by the eigenvalues of the matrix $M$

$$
M=\left(\begin{array}{cccc}
E_{\mathrm{C}}-i \gamma_{\mathrm{C}} & V_{1} & V_{2} & V_{3} \\
V_{1} & E_{\mathrm{X} 1}-i \gamma_{1} & 0 & 0 \\
V_{2} & 0 & E_{\mathrm{X} 2}-i \gamma_{2} & 0 \\
V_{3} & 0 & 0 & E_{\mathrm{X} 3}-i \gamma_{3}
\end{array}\right) .
$$

The coupling strength $V_{\mathrm{i}}$ couples the excitonic modes $E_{\mathrm{Xi}}$ with homogeneous linewidths $\gamma_{\mathrm{i}}$ to the cavity mode $E_{\mathrm{C}}$ with homogeneous linewidth $\gamma_{\mathrm{C}}$. For systems with less than three excitons, $N<3$, the eigenvalue problem was reduced to solving a matrix of the order of $N+1$ by setting $E_{\mathrm{Xi}}, \gamma_{\mathrm{i}}$, and $V_{\mathrm{i}}$ to zero for all excitons with indices $i$ greater than $N$.

In our experiment, we tested different thicknesses $d_{\text {org }}$ of the organic material. At points of zero detuning, i.e., when $E_{\mathrm{X}}=E_{\mathrm{C}}$, one expects a minimum mode splitting (Rabi splitting) of

$$
\hbar \Omega_{\mathrm{R}, \mathrm{i}}=2 \sqrt{V_{\mathrm{i}}^{2}-\left(\frac{\gamma_{\mathrm{i}}-\gamma_{\mathrm{C}}}{2}\right)^{2}} .
$$

The homogeneous linewidth of the excitons can be estimated from the exciton lifetimes (several hundreds of picoseconds, see Table I) to be of the order of $\gamma_{\mathrm{i}} \lesssim 10^{-6} \mathrm{eV},{ }^{21,22}$ whereas the linewidths of the investigated microcavities were determined by $\mathrm{TM}$ simulations to be $\gamma_{\mathrm{C}, \mathrm{Al}}=0.2 \mathrm{eV}$ and $\gamma_{\mathrm{C}, \mathrm{Ag}}=0.05 \mathrm{eV}$. Thus, the coupling strength is expected to be mainly limited by the lifetime of the cavity photon. However, even the influence of $\gamma_{\mathrm{C}}$ on the Rabi splitting is small; comparisons of $\mathrm{CO}$ calculation taking into account and not taking into account $\gamma_{\mathrm{C}}$ only showed differences of $\left(\hbar \Omega_{\gamma c=0}-\hbar \Omega_{\gamma c \neq 0}\right)<0.05 \hbar \Omega_{\gamma c=0}$. Hence, the calculations presented below assume $\gamma_{\mathrm{i}}=\gamma_{\mathrm{C}}=0$ to keep the number of fitting parameters in the model as small as possible. Consequently, the Rabi splitting is simply deduced from Equation (2) as $\hbar \Omega_{\mathrm{R}, \mathrm{i}}=2 V_{\mathrm{i}}$.

Figure 4 shows the reflectance of our cavities as a function of energy and organic film thickness. The figure compares TM calculations (grey background) to the dips observed in the experimental data (open symbols) and to the eigenenergies obtained from the CO model (solid lines). The summary of theoretically and experimentally obtained data in one graph demonstrates that for all studied organic materials, the cavity modes show clear anticrossing of the excitonic and bare cavity resonances, which is the evidence of strong exciton-photon coupling. In PF8, F8BT, and BBEHP-PPV, the modes at large thicknesses show strong coupling of the exciton with the second lowest photon mode of the cavity. In order to minimise the number of fitting parameters and keep the analytical model as simple as possible, different optical modes were assumed to couple with the same constant to an exciton. For PF8 and F8BT, we observe a strong deviation between $\mathrm{CO}$ and TM calculations if we do not include the absorption in the UV (the energy of these UV excitons is too high to be marked in Figure 4), which is why the highest energy polariton branches observed correspond to middle

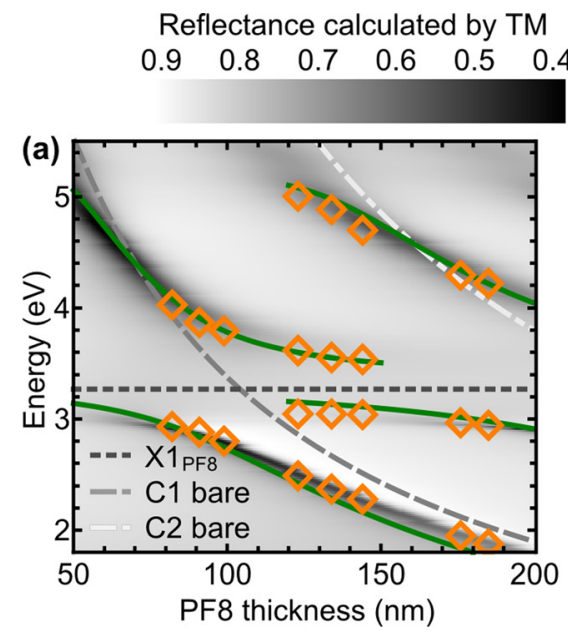

$\triangle$ experimental modes
modes from $\mathrm{CO}$
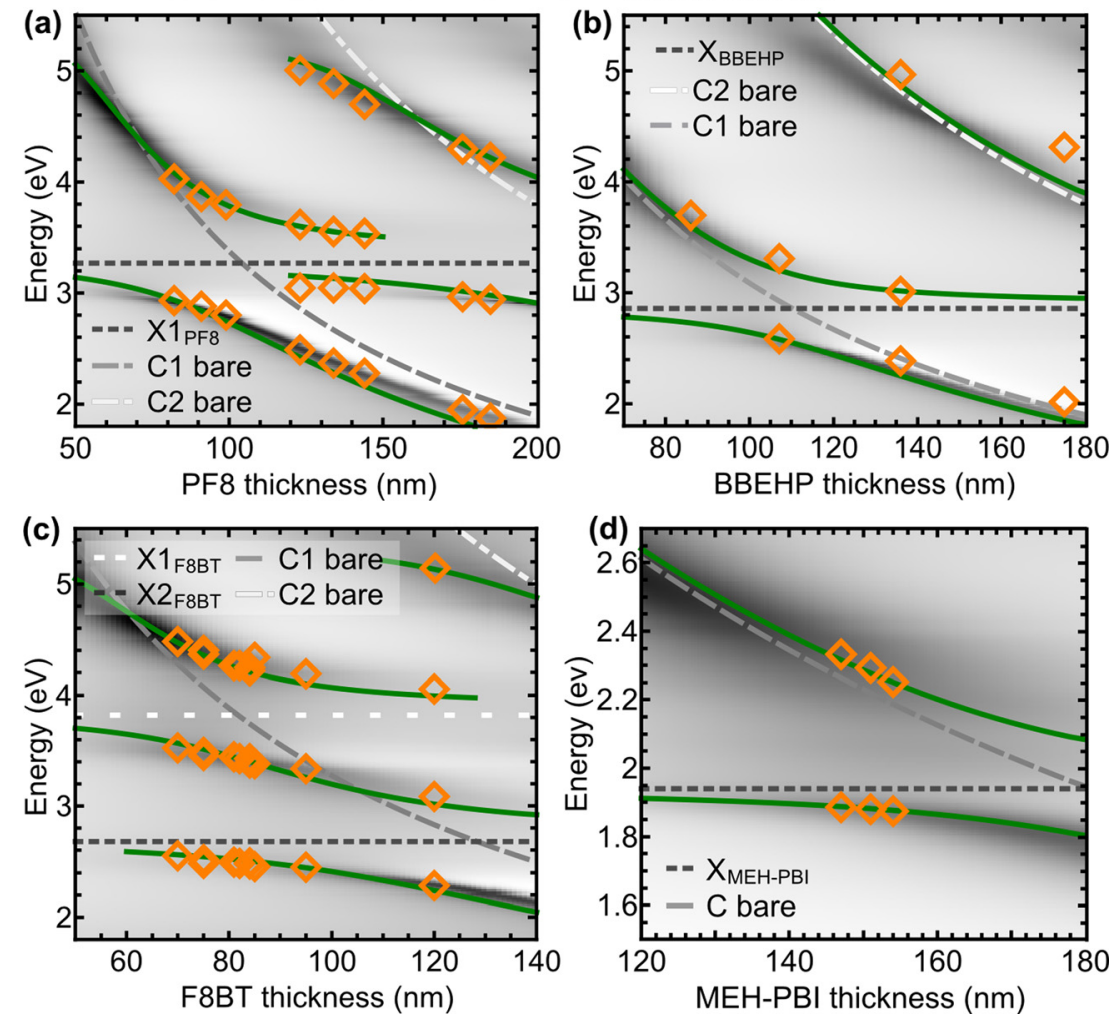

FIG. 4. Comparison of the experimental reflection minima (open symbols, material thickness determined by transfer matrix simulations) to transfer matrix calculations of the cavity reflectance (background) and to the position of the polariton modes as determined by a coupled oscillator model (solid lines), as well as the spectral position of the bare cavity and excitonic mode (dashed lines). (a)-(d) Data for different organic materials: (a) PF8, (b) BBEHPPPV, (c) F8BT, and (d) MEH-PBI. 
polaritons and not to upper polaritons (UP). The modes of the F8BT-filled cavity split into three different branches, as expected for a material with two pronounced absorption resonances within the investigated spectral range.

By modelling our system as a two- to four-level coupled oscillator model, we simplify its description significantly: in addition to the more complex exciton density of states of the real system, the approximation of the cavity mode neglects effects like the potential change of the refractive index with varying film thicknesses due to density variation and assumes a spectrally uniform effective refractive index $n_{\text {eff }}(E)=$ const. Nevertheless, the remarkable agreement with the experimental data confirms both the TM and the $\mathrm{CO}$ model and also justifies the approximations used.

In order to quantify the coupling strength, we extracted the Rabi splitting from the $\mathrm{CO}$ and the TM calculations. For the CO calculations, $\hbar \Omega_{\mathrm{R}}$ is obtained directly from the fitting parameter $V_{\mathrm{i}}$ (using Equation (2) with $\gamma_{\mathrm{C}}=\gamma_{\mathrm{i}}=0$ ). From the TM calculations, $\hbar \Omega_{\mathrm{R}}$ is determined as the smallest distance between the two reflectance minima corresponding to LP and UP when analysing the spectra over the relevant thickness range. For each material, the mean of the determined $\hbar \Omega_{\mathrm{R}}$ values is listed in Table I. The differences between the Rabi splittings determined by the two methods were smaller than the given uncertainty of $\pm 0.05 \mathrm{eV}$, which also estimates the tolerance of the $\mathrm{CO}$ fits since these do not match perfectly with the $\mathrm{CO}$ calculation over the entire thickness range. The table compares $\hbar \Omega_{R}$ to the material parameters discussed above, from which a correlation between $\hbar \Omega_{\mathrm{R}}$ and the absorption characteristics is evident, consistent with our earlier observations for fluorescent proteins. ${ }^{28}$ This dependence appears similarly for $\alpha_{\max }$ and $\alpha_{\text {int }}$. In contrast, no direct connection of $\hbar \Omega_{R}$ to the other parameters listed in the table can be found. This holds also for the excitonic linewidth, the exciton lifetime, and $\Delta_{\text {abs- }}$ $\mathrm{em}$, which are all parameters often thought to be important for achieving large coupling strengths. ${ }^{6,16-20}$ We believe that this statement can likely be generalised, at least to other organic emitters, as it holds not only for materials with similar chemical characteristics (i.e., the two polyfluorene polymers studied) but also for the distinct J-aggregate MEH-PBI.

In conclusion, we found clear signatures of strong exciton-photon coupling in metal-clad microcavities for the four investigated organic materials PF8, BBEHP-PPV, F8BT, and MEH-PBI. The importance of taking into account the optical anisotropy of the analysed materials, which originates from their different ordering in the thin film, was demonstrated. A comparison of the Rabi splitting to various optical properties of the compounds emphasised, in agreement with expectations, the role of the absorption as an important parameter for the coupling strength of the material leading to a record Rabi splitting observed in organic microcavities of $1.09 \mathrm{eV}$. Linking the two observations, we deduce that the preferential orientation of transition dipoles in the plane, as seen in all investigated materials, enhances the coupling strength compared to isotropically oriented dipoles due to a more efficient coupling to the cavity photons.

The authors thank J. Keeling and G. A. Turnbull for fruitful discussions. We acknowledge financial support from the European Research Council (ERC StG 640012), the Scottish Funding Council (through SUPA), the studentship funding through the EPSRC CM-CDT (EP/L015110/1), and the EPSRC Hybrid Polaritonics program grant (EP/M025330/ 1). I. D. W. Samuel and P. J. Skabara each acknowledge support from a Royal Society Wolfson Research Merit Award. A. L. Kanibolotsky thanks the EPSRC for funding (EP/I029141). The research data which is presented in this publication is publicly available at http://dx.doi.org/ 10.17630/a78ef6e6-82ac-401d-8b00-cead26f56190.

${ }^{1}$ C. Weisbuch, M. Nishioka, A. Ishikawa, and Y. Arakawa, Phys. Rev. Lett. 69, 3314 (1992).

${ }^{2}$ J. Kasprzak, M. Richard, S. Kundermann, A. Baas, P. Jeambrun, J. M. J. Keeling, F. M. Marchetti, M. H. Szymańska, R. André, J. L. Staehli, V. Savona, P. B. Littlewood, B. Deveaud, and L. S. Dang, Nature 443, 409 (2006).

${ }^{3}$ G. Christmann, R. Butté, E. Feltin, J.-F. Carlin, and N. Grandjean, Appl. Phys. Lett. 93, 051102 (2008).

${ }^{4}$ K. S. Daskalakis, P. S. Eldridge, G. Christmann, E. Trichas, R. Murray, E. Iliopoulos, E. Monroy, N. T. Pelekanos, J. J. Baumberg, and P. G. Savvidis, Appl. Phys. Lett. 102, 101113 (2013).

${ }^{5}$ K. S. Daskalakis, S. A. Maier, R. Murray, and S. Kéna-Cohen, Nat. Mater. 13, 271 (2014).

${ }^{6}$ J. D. Plumhof, T. Stöferle, L. Mai, U. Scherf, and R. F. Mahrt, Nat. Mater. 13, 247 (2013).

${ }^{7}$ A. Baas, J. Karr, H. Eleuch, and E. Giacobino, Phys. Rev. A 69, 023809 (2004).

${ }^{8}$ A. Biswas and D. Milovic, Commun. Nonlinear Sci. Numer. Simul. 15, 1473 (2010).

${ }^{9}$ A. Amo, S. Pigeon, D. Sanvitto, V. G. Sala, R. Hivet, I. Carusotto, F. Pisanello, G. Lemenager, R. Houdre, E. Giacobino, C. Ciuti, and A. Bramati, Science 332, 1167 (2011).

${ }^{10}$ B. Eiermann, T. Anker, M. Albiez, M. Taglieber, P. Treutlein, K.-P. Marzlin, and M. Oberthaler, Phys. Rev. Lett. 92, 230401 (2004).

${ }^{11}$ M. Sich, D. N. Krizhanovskii, M. S. Skolnick, A. V. Gorbach, R. Hartley, D. V. Skryabin, E. A. Cerda-Méndez, K. Biermann, R. Hey, and P. V. Santos, Nat. Photonics 6, 50 (2011).

${ }^{12}$ A. Imamoglu, R. Ram, S. Pau, and Y. Yamamoto, Phys. Rev. A 53, 4250 (1996).

${ }^{13}$ D. S. Lidzey, D. D. C. Bradley, M. S. Skolnick, T. Virgili, S. Walker, and D. M. Whittaker, Nature 395, 53 (1998).

${ }^{14}$ J. Tischler, M. Bradley, V. Bulović, J. Song, and A. Nurmikko, Phys. Rev. Lett. 95, 036401 (2005).

${ }^{15}$ S. Kéna-Cohen and S. R. Forrest, Nat. Photonics 4, 371 (2010).

${ }^{16}$ D. M. Coles, N. Somaschi, P. Michetti, C. Clark, P. G. Lagoudakis, P. G. Savvidis, and D. G. Lidzey, Nat. Mater. 13, 712 (2014).

${ }^{17}$ R. J. Holmes and S. R. Forrest, Phys. Rev. Lett. 93, 186404 (2004).

${ }^{18}$ J. Wenus, L. G. Connolly, and D. G. Lidzey, Phys. Status Solidi (c) 2, 3899 (2005)

${ }^{19}$ D. M. Coles, P. Michetti, C. Clark, A. M. Adawi, and D. G. Lidzey, Phys. Rev. B 84, 205214 (2011).

${ }^{20}$ N. Christogiannis, N. Somaschi, P. Michetti, D. M. Coles, P. G. Savvidis, P. G. Lagoudakis, and D. G. Lidzey, Adv. Opt. Mater. 1, 503 (2013).

${ }^{21}$ A. Rose, Z. Zhu, C. F. Madigan, T. M. Swager, and V. Bulović, Nature 434, 876 (2005).

${ }^{22}$ R. Xia, G. Heliotis, Y. Hou, and D. D. Bradley, Org. Electron. 4, 165 (2003).

${ }^{23}$ S. Herbst, B. Soberats, P. Leowanawat, M. Lehmann, and F. Würthner, Angew. Chem. Int. Ed. 56, 2162 (2017).

${ }^{24}$ B. M. Landreth and S. I. Stupp, Macromolecules 20, 2083 (1987).

${ }^{25}$ M. Ariu, D. G. Lidzey, M. Sims, A. J. Cadby, P. A. Lane, and D. D. C. Bradley, J. Phys.: Condens. Matter 14, 9975 (2002).

${ }^{26}$ Y. Wang, P. O. Morawska, A. L. Kanibolotsky, P. J. Skabara, G. A. Turnbull, and I. D. W. Samuel, Laser Photonics Rev. 7, L71 (2013).

${ }^{27}$ C. P. Dietrich, A. Steude, L. Tropf, M. Schubert, N. M. Kronenberg, K. Ostermann, S. Höfling, and M. C. Gather, Sci. Adv. 2(8), e1600666 (2016).

${ }^{28}$ C. P. Dietrich, A. Steude, J. Ohmer, U. Fischer, S. Höfling, and M. C. Gather, Adv. Opt. Mater. 5, 1600659 (2017). 